\title{
A Photometric and Spectroscopic Study of Non-barred Ringed Galaxies
}

\author{
R. Grouchy and R. Buta \\ Dept. of Physics \& Astronomy, University of Alabama, Tuscaloosa, AL 35487, USA
}

\begin{abstract}
We present preliminary analysis of an imaging survey of non-barred ringed galaxies (NBRGs). We have taken broadband, narrow band, and spectroscopic data for a sample of NBRGs selected from the Third Reference Catalogue of Bright Galaxies and the Catalogue of Southern Ringed Galaxies. These galaxies are the subject of a survey in order to (1) better understand the mode of ring formation in the absence of a strong bar and (2) delineate the general photometric and spectroscopic properties of this group as a whole.
\end{abstract}

Keywords. Galaxies: Fundamental Properties, Galaxies: Structure, Galaxies: Evolution

\section{Introduction}

It is generally accepted that rings form through the accumulation of gas into orbits in resonance with a strong bar. Therefore, it is interesting to note that non-barred galaxies also show rings just as in barred galaxies. A study of this class could bring a better understanding of how disk galaxies form and evolve through time. For example, perhaps NBRGs were once strongly barred, creating the ring feature and have since evolved out of a bar phase. The data will be used to (1) characterize the appearance and placement of the rings and compare them to (a) other NBRGs and (b) barred galaxies, (2) study color distribution and star formation properties, (3) detect weak possibly fading bars in longer wavelengths, (4) detect secondary/misaligned bars, which is a possible marker of a destroyed bar (Friedli 1996), and (5) measure underlying bulge and disk structure. The sample includes ESO 297-27, which displays rare counter winding spiral structure, in which two sets of non-overlapping spiral arms appear to unwind in opposite directions. This unusual structure, so far, has been seen only in NBRGs (Buta et al. 2003). This work was supported by NSF Grant AST 050-7140 to the University of Alabama.

\section{Future developments}

In the future, we will attempt to recreate the galaxies using a computer simulation (Bournaud 2002) which evolves a galaxy through time either on its own, by accumulating cold gas, or by perturbations from a flyby companion. We also plan to use models of minor mergers and a model of close approach (Byrd et al. 1994) to attempt to recreate the galaxies. Due to the wide dispersion in metric and photometric properties of this group of galaxies, the process of ring formation will also be varied.

\section{References}

Bournaud, F. \& Combes, F. 2002, A\&A 392, 83.

Buta, R., Byrd, G., \& Freeman, T. 2003, AJ 125, 634.

Byrd, G., Freeman, T., \& Howard, S. 1994, AJ 108, 2078.

Friedli, D. 1996, A\&SA, 312, 761. 